\title{
Combined Lowering Effects of Rosuvastatin and L. acidophilus on Cholesterol Levels in Rat
}

\author{
Lijun Wang ${ }^{1,2 *}$, Baihua Zhou ${ }^{1}$, Xue Zhou ${ }^{3}$, Yang Wang ${ }^{2}$, Hongwei Wang ${ }^{1}$, Shengying Jia ${ }^{1}$, Zhipeng Zhang ${ }^{1}$, \\ Chao $\mathrm{Chu}^{2}$, and Jianjun $\mathrm{Mu}^{2 *}$ \\ ${ }^{1}$ Department of Cardiology, Affiliated Zhongshan Hospital, Dalian University, Dalian 116001, P.R. China \\ ${ }^{2}$ Department of Cardiology, The First Affiliated Hospital of Xi'an Jiaotong University, Xi'an 710061, P.R. China \\ ${ }^{3}$ Department of Laboratory, The Second Affiliated Hospital of Jiaxing University, Jiaxing Second Hospital, Jiaxing 314000, P.R. China
}

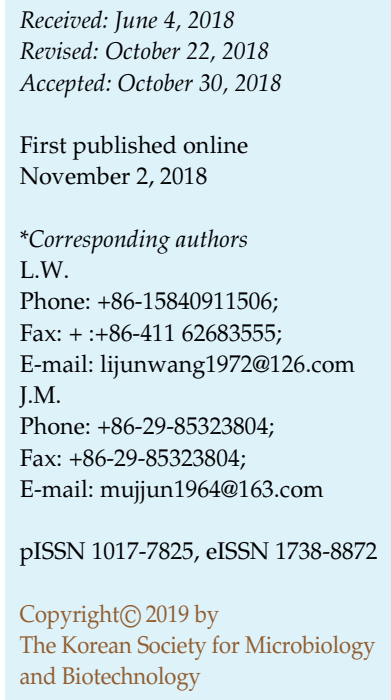

Statins are a class of lipid-lowering drugs commonly used in the prevention of cardiovascular diseases. However, statin therapy presents many limitations, which have led to an increased interest in non-drug therapies, such as probiotics, to improve blood cholesterol levels. Indeed, probiotic strains such as Lactobacillus acidophilus have been found to improve blood lipid profiles, especially in reducing total cholesterol and LDL-C levels. In this study, we established a high-cholesterol rat model and studied the effect of Lactobacillus acidophilus administration alone or in combination with rosuvastatin. We were able to show that Lactobacillus exerts a cholesterol-lowering effect. Additionally, we observed that when administered together, rosuvastin and Lactobacillus exert a combined cholesterol-lowering effect. Altogether, our data advocate for the possibility of establishing probiotics as non-drug supplements for the treatment of hypercholesterolemia.

Keywords: Lactobacillus acidophilus, rosuvastatin, cholesterol, hypercholesterolemia, cardiovascular disease

\section{Introduction}

The causal link between hypercholesterolemia and cardiovascular diseases (CVD) is well established [1]. Achieving lower blood lipids and blood cholesterol levels proved to be an efficient strategy in order to reduce atherosclerotic cardio vascular risk. In accordance with this strategy, statins, a group of lipid-lowering drugs, have been used routinely to treat hypercholesterolemic patients in the prevention of cardio vascular diseases [3]. Rosuvastatin is one of the most potent statins available and is effective even for patients presenting a high cardiovascular risk [4].

However, statin therapy presents many limitations. For example, only a fraction of the patients (approximately $20 \%$ ) achieve the therapeutic lipid-lowering target [5-7]. In addition, high doses of statin are associated with higher risk of hepatic and muscular side effects. Additionally, patients of Asian ethnicity are more susceptible to experience side effects due to genetic variations in hepatic metabolism and drug catabolism [8,9], especially at higher statin doses. Accordingly, those patients are usually prescribed lower doses [10]. Finally, adherence to statin therapy is generally poor [11]. Suboptimal adherence to statin therapy is a major barrier to achieving LDL-C targets [12] and statin down-titration or discontinuation occurs more frequently among patients with statin intolerance. These limitations, that cause a discrepancy between patients' requirements and clinical practice, have led to an increased interest in non-drug therapies to improve blood cholesterol levels.

Therapeutic intervention is not the only way to normalize cholesterol levels. Similar results can be obtained through simple lifestyle changes [13], such as regular physical activity [14-17], as well as adopting a specific diet [18-22]. The use of probiotics has also been shown to improve outcomes in patients with coronary heart disease with very low risk of side effects. Probiotics are microorganisms that 
bear various health benefits for the host upon consumption such as elimination of pathogens, alleviation of lactose intolerance, decreased serum cholesterol levels, reduced risk of cancer and antihypertensive effects [23]. Many probiotic strains such as Lactobacillus (L.) acidophilus, L. plantarum, L. casei, Bifidobacterium longum, Enterococcus faecium, and Streptococcus thermophilus have been found to improve blood lipid profiles, especially in reducing total cholesterol and LDL-C levels [24]. These observations advocate for the possibility of establishing probiotics as non-drug supplements for hypercholesterolemia.

To the best of our knowledge, to date, no study has explored the efficacy of a treatment of hypercholesterolemia combining the use of rosuvastatin and probiotics. The aim of this work is to fill this gap by determining whether supplementing rosuvastatin (sp) therapy with the administration of L. acidophilus can result in improved lipidlowering effects.

\section{Material and Methods}

\section{Bacterial Cultures}

L. acidophilus ATCC 4356 strain was chosen based on its demonstrated hypocholesterolemic effect both in vitro [25] and in vivo $[26,27]$. The strain was obtained from ATCC (American Type Culture Collection, USA). The organisms were activated successively three times in sterile de Man, Rogosa, and Sharpe (MRS) broth (Hi-Media, India) before experimental use. L. acidophilus was cultured anaerobically for 24 to $36 \mathrm{~h}$ at $37^{\circ} \mathrm{C}$ until the OD value reached 3-4. L. acidophilus cells were subsequently separated from the medium through centrifugation $(600 \mathrm{~g}, 10 \mathrm{~min})$. The supernatant was discarded and PBS was used to carefully resuspend the cells.

\section{Animals, Diets, and Sample Collection}

Forty specific-pathogen-free (SPF) Sprague Dawley (SD) male rats $(200 \pm 20 \mathrm{~g})$ were purchased from Dalian Medical University Laboratory Animal Center (application license No.: SYXK (Liao)2013-0006). The rats were housed in stainless steel cages, in a temperature controlled room having 12 hourly light and dark cycles, at temperatures between $24-26^{\circ} \mathrm{C}$ and a relative humidity of $55-60 \%$. The rats were offered food and water ad libitum. All animal experiments were approved by, and performed in accordance with the Institutional Animal Care and Use Committee of Dalian Medical University (Dalian, Liaoning, China).

Rats were fed with high-fat diet (HFD), containing $87.8 \%$ ordinary feed, $10 \%$ lard, $2 \%$ cholesterol, and $0.2 \%$ propylthiouracil, for 4 weeks. Upon induction of hypercholesterolemia, where serum cholesterol levels doubled in comparison to their initial serum cholesterol levels, they were then divided randomly into four groups of ten rats each: (A) the HFD control group, $2 \mathrm{ml} / \mathrm{d}$ phosphate buffer saline (PBS; $0.01 \mathrm{M} ; \mathrm{pH} 7.4$ ); (B) the rosuvastatin group, $2 \mathrm{ml} / \mathrm{d}$ PBS containing rosuvastatin (AstraZeneca Pharmaceutical Co., Ltd. SFDA approval No. J20120006) (10 mg/kg) (C) the L. acidophilus group, $2 \mathrm{ml} / \mathrm{d}$ PBS containing L. acidophilus at the standard concentration of $10^{9} \mathrm{CFU} / \mathrm{ml}$ [28-30]; and (D) the rosuvastatin + L. acidophilus group, $2 \mathrm{ml}$ PBS containing rosuvastatin (10 $\mathrm{mg} / \mathrm{kg}$ and L. acidophilus $10^{9} \mathrm{cfu} / \mathrm{ml}$ ). All four groups received their respective PBS solution intragastrically, and all groups had free access to HFD and water ad libitum for four weeks. Body weight and amount of feed intake were recorded weekly.

\section{Blood and Stool Sample Collection}

After two weeks and four weeks, the drug was withdrawn. After fasting for $12 \mathrm{~h}$, the blood samples were obtained from the orbital venous plexus. Serum and red blood cells (RBC) were separated by centrifugation at $5,000 \mathrm{rpm}$ for $10 \mathrm{~min}$ at $4^{\circ} \mathrm{C}$. All serum samples were then snap-frozen in liquid nitrogen prior to storage at $-80^{\circ} \mathrm{C}$. The serum total cholesterol (TC), low-density lipoprotein cholesterol (LDL-C), high-density lipoprotein cholesterol (HDL-C) and C-reactive protein (CRP) were detected by using commercial kits (Ningbo Meikang Biotechnology Co., Ltd., China) according to the manufacturer's instructions. Feces were collected from each group before treatment (week 0) and two weeks after treatment and stored at $-80^{\circ} \mathrm{C}$ until further analysis. The fecal contents were taken after four weeks (day 29) under aseptic conditions.

\section{Microbial DNA Extraction from Feces}

Total bacterial DNA was extracted from $250 \mathrm{mg}$ of fecal sample using QIAamp DNA Stool Mini Kit (QIAGEN, UK) with modified protocol [31]. The DNA samples were resuspended in $100 \mathrm{ul}$ of TE buffer and treated with $2 \mathrm{ul}$ of DNase-free RNase $(10 \mathrm{mg} / \mathrm{ml})$ at $37^{\circ} \mathrm{C}$ for $15 \mathrm{~min}$. Proteins were removed by treatment with $15 \mathrm{ul}$ of proteinase $\mathrm{K}$ at $70^{\circ} \mathrm{C}$ for $10 \mathrm{~min}$. DNA was subsequently purified using QIAamp Mini Spin columns (QIAGEN) following the manufacturer's instructions. Final DNA concentration was quantified by using NanoDrop ND-1000 (NanoDrop Technologies, $\mathrm{DE})$.

\section{Fecal Microbiota Composition}

Feces samples were freeze-dried overnight prior to DNA extraction as previously described [32]. The V3-V4 region of the $16 \mathrm{~S}$ rRNA gene was amplified using the primers 338F (5'ACTCCTACGGRAGGCAGCAG-3') and 806R (5'-GGACTACHV GGGTWTCTAAT-3'). PCR products were purified using AMPure $\mathrm{XP}$ magnetic purification beads (Beckman Coulter, Inc., Brea, USA) and quantified using the Quant-iT PicoGreen dsDNA Assay Kit (Life Technologies Japan, Ltd, Japan). 16S rRNA sequencing (Roche Applied Science, Indianapolis, Indiana) was performed using 454 GS JUNIOR according to the manufacturer's instructions. The resulting 16S rRNA reads were analyzed using the QIIME pipeline [33]. Taxonomic assignments and estimation of relative abundance of sequencing data were performed using 
the analysis pipeline of the QIIME software package. Operational taxonomic units (OTUs) were taxonomically classified based on comparison with the Greengenes database using the RDPclassifier [34, 35]. Unifrac distance calculations were done using the $\mathrm{R}$ software package ( $\mathrm{R}$ Development Core Team, Vienna, Austria (https://www.r-project.org).

\section{Statistical Analysis}

Data were subjected to one-way analysis of variance (ANOVA) using the Statistical Package for the Social Science (SPSS 19.0, SPSS, Inc., USA), and the Mann-Whitney $U$ test was used to compare the differences among various groups. Differences were considered significant when the probability was less than 0.05 . All data were expressed as the mean \pm standard error of means, with $n=10$ being the number of samples for each group.

\section{Results and Discussion}

\section{Generation of Hypercholesterolemic Rats}

All rats appeared healthy throughout the study; the body weight gain, feed intake, and feed efficiency were stable during the normal diet period. After being fed the HFD for four weeks, the animals exhibited slightly higher body weights and significantly higher TC, LDL-C and HDL-C levels in serum $(p<0.001)$ showing that the HFD indeed induced hypercholesterolemia (Fig. 1).

\section{Lipid Profiles}

After four weeks of HFD, the rats were subjected to four weeks of therapy trial while maintaining a high fat diet. The levels of cholesterol, including TC, HDL-C, and LDL-C in the serum of all rats were measured after two weeks and

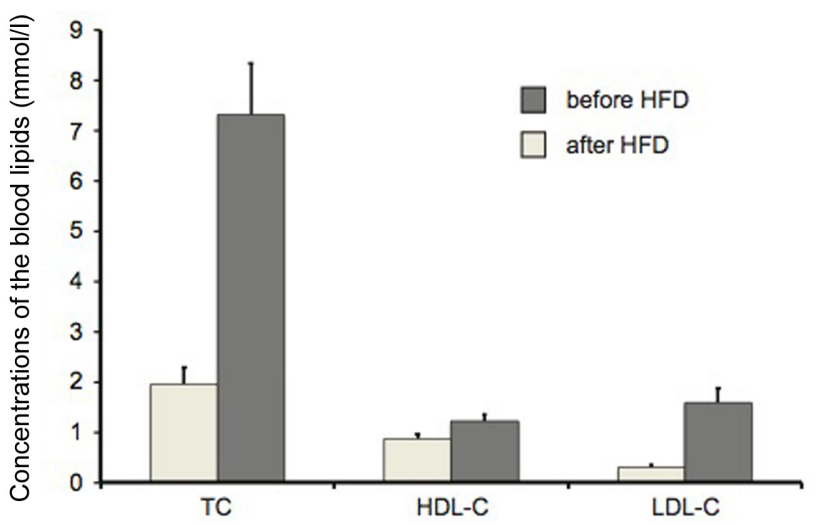

Fig. 1. Serum TC, HDL-C, and LDL-C concentrations of hypercholesterolemia-induced rats before and after fourweek HFD feeding.

Error bars represent standard error of means; $n=40, p<0.05$. four weeks of therapy (Figs. 2A and 2B). The concentration of serum TC for rats in the HFD control group (no treatment, group A) was higher than for those in all other treatment groups $(p<0.05)$. Rosuvastatin at a dose of $10 \mathrm{mg} / \mathrm{kg}$ (group B) decreased TC concentrations by 15.6\% (after two weeks) and $12.8 \%$ (after four weeks), when compared to the HFD-control (group A) $(p<0.05)$. L. acidophilus at doses of $10^{9} \mathrm{CFU} / \mathrm{ml}, 2 \mathrm{ml} /$ day (group C) decreased TC concentrations by $20.1 \%$ (after two weeks) and $24.8 \%$ (after four weeks), when compared to the HFD-control (group A) $(p<0.05)$. The combination of those 2 treatments together (group D) further decreased the TC concentration by $25.6 \%$ (after two weeks) and $31.8 \%$ (after four-weeks). The HDL-C concentrations for rats in all groups remained at similar levels, except for the group with combined treatments showing higher HDL-C levels compared to the control and rosuvastatin groups, although this difference does not
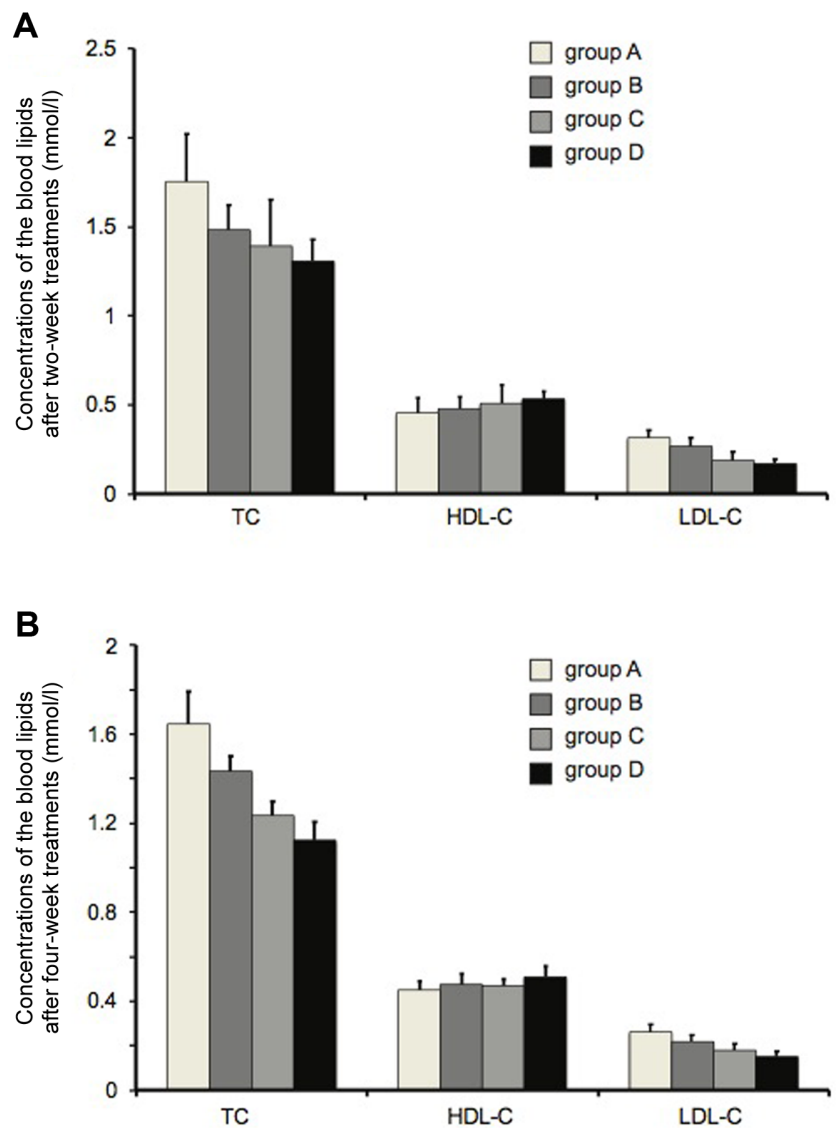

Fig. 2. Serum TC, HDL-C, and LDL-C concentrations of hypercholesterolemia-induced rats after two weeks (A) and four weeks (B) of treatment.

Error bars represent standard error of means; $n=10$ for each group, $p<0.05$. 
appear to be statistically significant. After two weeks of treatment, LDL-C levels of hypercholesterolemic rats administered with L. acidophilus at doses of $10^{9} \mathrm{CFU} / \mathrm{ml}$, $2 \mathrm{ml} /$ day (group C) and combined treatments (group D) were, however, significantly lower than that of the HFD control group by $39.7 \%$ and $46.1 \%$, respectively $(p<0.05)$. Surprisingly, the hypocholesterolemic effect of L. acidophilus was found to be better than that of rosuvastatin, at least under this experimental setup.

\section{C-Reactive Proteins (CRP) and Arterial Stiffness Index (ASI) Profiles}

To assess the risk of coronary heart disease (CHD) of these hypercholesterolemic rats, we also monitored CRP and ASI levels (Fig. 3). The groups with treatments compared to the control group experienced a decrease of CRP levels $(p<0.05)$. The changes after 4 weeks of treatment in CRP levels were similar between the combination (D), L. acidophilus (C) and rosuvastatin (B) groups. The ASI, which is an indicator of arterial stiffness, is significantly lower for all the groups with drug and/or probiotic treatments when
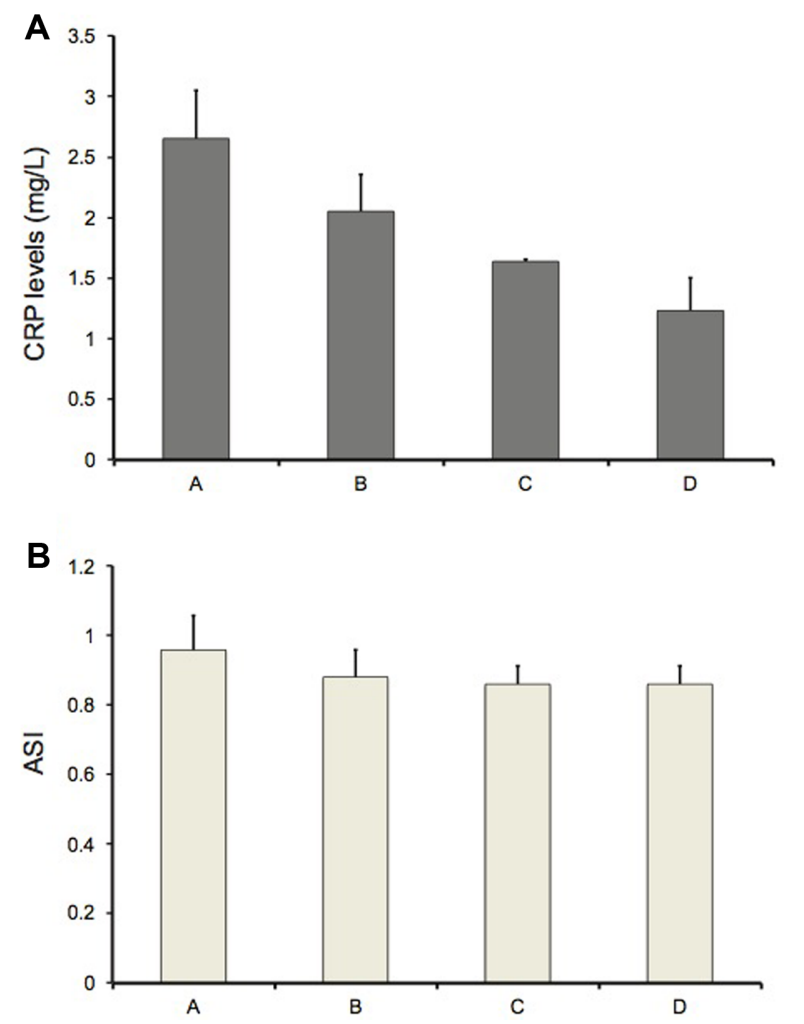

Fig. 3. CRP (A) and ASI (B) levels of hypercholesterolemiainduced rats after four weeks of treatment.

Error bars represent standard error of means; $n=10$ for each group, $p<0.05$. compared to the control group $(p<0.01)$. Furthermore the ASI for the L. acidophilus group was lower than for the rosuvastatin group and the ASI for the combination treatment group was even lower than for the L. acidophilus alone group $(p<0.01)$.

\section{Intestinal Microbiota Composition}

Fecal microbiota composition of 3 randomly selected animals in each group was analyzed by $16 \mathrm{~S}$ rRNA sequencing of fecal samples. A stacked bar chart showing the microbiota composition for the rosuvastatin, the L. acidophilus alone, the combination and the control groups after the four-week therapy trial is shown in Fig. 4A. Data showed that there was an increase in Lactobacillus

A genustree

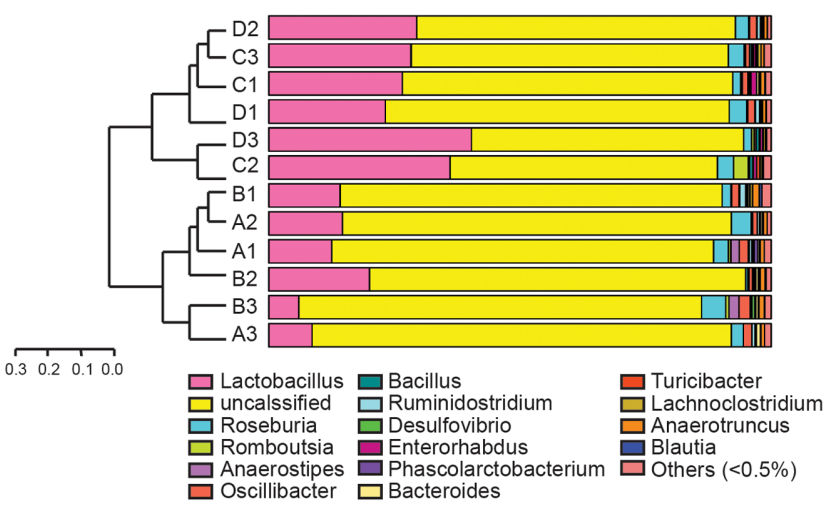

B

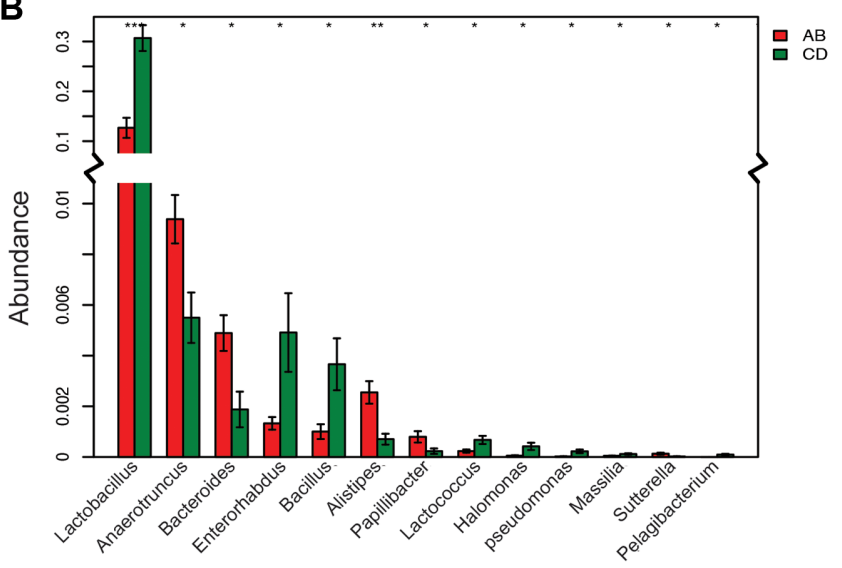

Fig. 4. Intestinal microbiota composition of all the groups. (A) Fecal microbiome structure variation among groups A, B, C, and D. Each of the stacked bar plots illustrates the relative abundance of the fecal microbiota at the genus levels for each of the individuals. (B) Analysis of the level of genus difference between group $\mathrm{AB}$ and $\mathrm{CD}$ shown by the anova one-way method at four weeks of treatment. 
population for both the L. acidophilus and the combination groups. The bray method was used to calculate the difference in abundance of the genus population between the samples. Groups A and B were clustered as there was no significant difference in the species structure between these samples. The groups C and D were clustered as well as they also display similar structures.

The one-way analysis of variance (ANOVA) analysis showed that Lactobacillus in cluster $\mathrm{CD}$ is higher than that in cluster $A B$ revealing the differences generated by the administration of L. acidophilus (Fig. 4B).

\section{Dietary Influence on Hypercholesterolemic Rats}

Previous studies have suggested that the fat and cholesterol levels in diet are highly correlated with the cholesterol concentrations in blood [36, 37]. Indeed, blood cholesterol has both an endogenous and a dietary origin through the reabsorption of biliary cholesterol in the small intestine [38]. Since we are interested in exploring the nondrug therapies complementary to rovustatins, we also conducted a feeding trial to analyze the effects of a change of diet on hypercholesterolemic rats. After 2 weeks on a normal diet, we observed that the blood levels of TC, LDL$\mathrm{C}$, and HDL-C were significantly lower. After 4 weeks, the risk of hypercholesterolemia continued to decrease as shown by a composite score reflecting the variations in TC, LDL-C, and HDL-C $(p<0.01)$ (Fig. 5). Thus, daily diet has a concurrent and clinically significant effect on the blood cholesterol levels of hypercholesterolemic rats.

A number of studies have shown that beneficial bacterial additives, by changing the composition of the intestinal

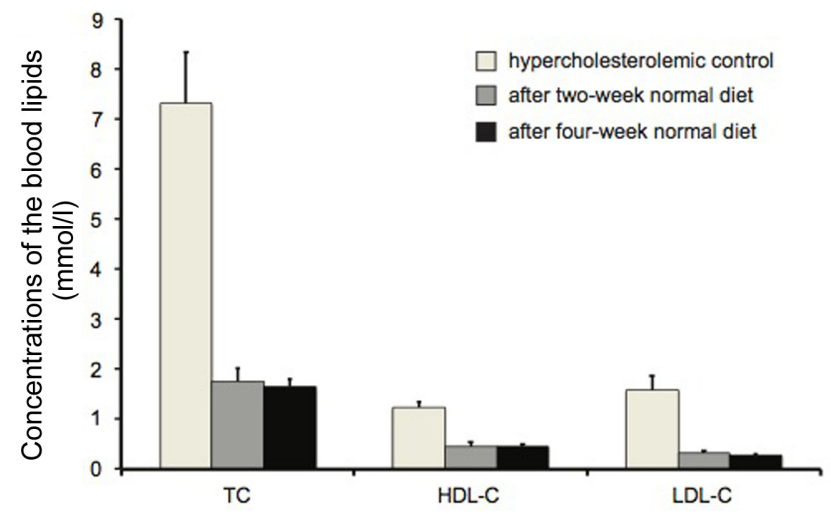

Fig. 5. Serum TC, HDL-C, and LDL-C concentrations of hypercholesterolemia-induced rats after two weeks and four weeks of normal diet.

Error bars represent standard error of means; $n=10$ for each group, $p<0.01$. microflora and increasing the intestinal population of Lactobacillus, Bifidobacterium can help to lower cholesterol blood level [39-41]. Xie et al. fed rats with a high cholesterol diet, Lactobacillus acidophilus M1-16 and Lactobacillus plantarum 9-41-A for 6 weeks. The results showed that the number of colonies of Lactobacillus intestinalis and Bifidobacterium increased while the number of $E$. coli colonies decreased and that TC, LDL-C and TG levels were significantly reduced [42]. In a report where ezetimibe and simvastatin were used to alter the composition of the intestinal microbiota and the expression level of genes involved in the metabolism of cholesterol in rats, analysis of the gut microbiota showed that ezetimibe did not change the total bacteria, but selectively increased the proportion of Lactobacillus. Additionally, the levels of Lactobacillus in the ezetimibe or ezetimibe-plus-simvastatin group were negatively correlated with the expression of cholesterol metabolism-related genes, showing that ezetimibe exerts lipid-regulating effects by regulating Lactobacillus [43]. To sum up, these reports indicate that Lactobacillus plays a significant role in cholesterol metabolism.

In this work, a high-cholesterol rat model was established, and Lactobacillus acidophilus ATCC 4356 was intragastrically administered as the lipid-lowering properties of this strain have been shown both in vitro [25] and in vivo [26, 27]. According to the volume of the fecal contents, the number of bacteria was calculated according to the rat $\left(10 \mathrm{~g} * 10^{9} / \mathrm{d}\right)$ for 4 weeks. Compared to the control group, hypercholesterolemic SD rats given L. acidophilus experienced a decrease in TC and LDL-C after 2 weeks of 20.1\% and 39.7\% respectively, indicating that the blood cholesterol was significantly reduced. Those observations are consistent with the previous reports showing that Lactobacillus exerts a cholesterol-lowering effect [44-46].

Illuminate Miseq PE300 16S rDNA sequencing technology (6S V3-V4) high-throughput sequencing analysis was applied to the DNA of intestinal microflora of 3 randomly selected animals in each group at the $4^{\text {th }}$ week of treatment to establish a taxonomic comparison and determine the species difference. The results showed that the Lactobacillus in groups C and D at the genus level was significantly higher than that for the A and B groups. Plasma TC and LDL-C levels in the $C$ and $D$ groups were also significantly lower than those in the A and B groups, and negatively correlated with the levels of Lactobacillus in the intestinal tract, similar to previously reported results [47]. Other genera also showed significant differences between the two groups. The abundance of Lactobacillus, Enterorhabdus, Bacillus, Lactococcus, Halomonas, Pseudomonas, Massilia, and 
Pelagibacterium was significantly increased in the CD group compared to the AB group. Meanwhile, the abundance of Anaerrotruncus, Bacteroides, Alistipes, Papillibacter, Sutterella significantly decreased $(p \leq 0.05)$. Interestingly, in addition to Lactobacillus, Bacillus also has a demonstrated effect on blood lipid differences [48].

Lactobacillus exerts a cholesterol-lowering effect through various possible mechanisms [49-54]: (a) through the reduction of the synthesis of cholesterol due to the inhibition of the enzyme HMGCR; (b) it interferes in the bile salt pathway: The bile salt hydrolase produced by Lactobacillus can catalyze the deconjugation of combined bile acids into free bile acids; bile acids are excreted from the feces due to the non-repeated absorption of free bile acids in the human large intestine, thereby promoting the synthesis of new bile acids from cholesterol and promoting cholesterol catabolism. (c) It increases liver LDL-R expression: LDL-R can remove blood cholesterol contained in LDL particles to reduce total cholesterol levels. (d) Cell absorption and assimilation: Lactobacillus can absorb cholesterol into its own bacterium and is then discharged with the feces. (e) Organic acid salt production: is involved in the lowering of plasma TC and LDL-C levels.

The combined effects of rosuvastatin and another factor have already been observed, as Fei et al. reported a synergic protective effect on Acute Myocardial Infarction (AMI) involving rosuvastatin together with the Vascular Endothelial Growth Factor A (VEGF-A) [55]. However, to the best of our knowledge, there has been no study on the combined cholesterol-lowering efficacy of Lactobacillus and rosuvastatin. Rosuvastatin is mainly transported by the anion transporter polypeptide (OATPIBl) into hepatocytes [56]. Therefore, its lipid-regulating effect mainly relies on the presence of the transporter OATP1B1 on the cell membrane. The expression and activity of OATPs are regulated by various factors such as endocrine hormones, inflammatory factors and drugs [57]. Additionally, the modulation of OATP by proinflammatory cytokines is pervasive. A number of studies have shown that alterations in Bifidobacteria and Lactobacillus can cause changes in TNF- $\alpha$ and IL-6 levels [58-61]. High lipid levels can have severs effects on the intestinal flora, and decrease significantly the proportion of beneficial bacteria such as Lactobacillus. The decrease of Lactobacillus leads to an overexpression of the inflammatory cytokines TNF- $\alpha$ and IL-6, which in turn inhibits the expression of OATP1B1. The down-regulation of OATP1B1 in hepatocytes prevents the uptake of rosuvastatin which in turn enhances its lipid-lowering properties. The results of our study showed the combined cholesterol-lowering effect of the combined administration of Lactobacillus acidophilus and rosuvastatin. Indeed, the combined effect of Lactobacillus and rosuvastatin was better than that of statins and Lactobacillus alone. We assume that the combined effect is due to the lowering effect of increased Lactobacillus population on the level of inflammatory cytokines such as IL-6, therefore upregulating the expression of OATP1B1 and promoting the uptake of statins by hepatocytes.

The cholesterol in the body is mainly derived from food and biosynthesis. Cholesterol in food is converted into cholesterol monomers in the intestine, absorbed into the lymphatic system after absorption by the small intestine, and then entered into the bloodstream from the lymphatic system in the form of chylomicrons with various apolipoproteins. In this work, TC and LDL-C levels increased significantly in SD rats by high-cholesterol intake for one month. After adjusting to dietary structure, cholesterol levels decreased significantly after 2 weeks of eating regular diets, among which TC decreased by $76.3 \%$, $p<0.0001$; LDL-C decreased by $80 \%, p<0.001$. It is suggested that the dietary structure has a great influence on serum cholesterol levels in SD rats. Martínez et al. studied the interactions between diet, gut microbial ecology and cholesterol in model hamsters with hypercholesterolemia and found that the grain sorghum lipid extract (GSL) and the intestinal microbiota changes are closely related, suggesting that the effect of diet on cholesterol metabolism is, to some extent, achieved through the influence of intestinal flora [62].

The results of this work revealed that L. acidophilus showed good lipid-regulating effects on hypercholesterolemic rats, either on its own or used together with rosuvastatin. Our study also raises important questions such as the role of L. acidophilus in improving arteriosclerosis, its specific mechanism of lipid-lowering effect and its long-term efficacy, tolerability and safety.

\section{Acknowledgments}

This work was supported by the National Natural Science Foundation of China No. 81600327, China Postdoctoral Science Foundation funded project (2018M631177), China Postdoctoral Special Science Foundation funded project (No. 2018T111075), Fundamental Research Funds for the Central Universities (Grant Number: xjj2018103), and the Clinical Research Award of the First Affiliated Hospital of Xi'an Jiaotong University No. XJTU1AF-CRF-2017-021. 


\section{Conflict of Interest}

The authors have no financial conflicts of interest to declare.

\section{References}

1. Townsend N, Nichols M, Scarborough P, Rayner M. 2015. Cardiovascular disease in Europe 2015: epidemiological update. Eur. Heart J. 36: 2673-2674.

2. Catapano AL, Graham I, De Backer G, Wiklund O, Chapman MJ, Drexel H, et al. 2016. [2016 ESC/EAS Guidelines for the Management of Dyslipidaemias]. Kardiol. Pol. 74: 1234-1318.

3. Stone NJ, Robinson JG, Lichtenstein A. 2014. N. Engl. J. Med. 370: 1957.

4. Cosin Sales J, Fuentes Jimenez FJ, Mantilla Morato T, Ruiz E, Becerra V, Aceituno S, et al. 2015. [Cost-effectiveness of rosuvastatin versus simvastatin, atorvastatin and pitavastatin in patients with high and very high cardiovascular risk in Spain]. Clin. Investig. Arterioscler. 27: 228-238.

5. Holecki M, Handzlik-Orlik G, Almgren-Rachtan A, Dulawa J, Chudek J. 2017. The decreased achievement of therapeutic goal in lipid lowering therapy in obese and diabetic patients in Poland. Pharmacol. Rep. 69: 6-12.

6. Kotseva K, Wood D, De Bacquer D, De Backer G, Ryden L, Jennings $C$, et al. 2016. EUROASPIRE IV: a european society of cardiology survey on the lifestyle, risk factor and therapeutic management of coronary patients from 24 european countries. Eur. J. Prev. Cardiol. 23: 636-648.

7. Valerio MG, Velayati A, Jain D, Aronow WS. 2016. Promising new therapies for the treatment of hypercholesterolemia. Expert. Opin. Biol. Ther. 16: 609-618.

8. Wang P. 2011. Statin dose in Asians: is pharmacogenetics relevant? Pharmacogenomics 12: 1605-1615.

9. Yasuda SU, Zhang L, Huang SM. 2008. The role of ethnicity in variability in response to drugs: focus on clinical pharmacology studies. Clin. Pharmacol. Ther. 84: 417-423.

10. Cardiometabolic Risk Working Group: Executive C, Leiter LA, Fitchett DH, Gilbert RE, Gupta M, Mancini GB, et al. 2011. Cardiometabolic risk in Canada: a detailed analysis and position paper by the cardiometabolic risk working group. Can. J. Cardiol. 27: e1-e33.

11. Guglielmi V, Bellia A, Pecchioli S, Della-Morte D, Parretti D, Cricelli I, et al. 2017. Effectiveness of adherence to lipid lowering therapy on LDL-cholesterol in patients with very high cardiovascular risk: a real-world evidence study in primary care. Atherosclerosis 263: 36-41.

12. Chodick G, Shalev V, Gerber Y, Heymann AD, Silber H, Simah V, et al. 2008. Long-term persistence with statin treatment in a not-for-profit health maintenance organization: a population-based retrospective cohort study in Israel. Clin.
Ther. 30: 2167-2179.

13. Eckel RH, Jakicic JM, Ard JD, de Jesus JM, Houston Miller N, Hubbard VS, et al. 2014. 2013 AHA/ACC guideline on lifestyle management to reduce cardiovascular risk: a report of the American college of cardiology/American heart association task force on practice guidelines. J. Am. Coll. Cardiol. 63: 2960-2984.

14. Dattilo AM, Kris-Etherton PM. 1992. Effects of weight reduction on blood lipids and lipoproteins: a meta-analysis. Am. J. Clin. Nutr. 56: 320-328.

15. Huffman KM, Hawk VH, Henes ST, Ocampo CI, Orenduff MC, Slentz CA, et al. 2012. Exercise effects on lipids in persons with varying dietary patterns-does diet matter if they exercise? Responses in studies of a targeted risk reduction intervention through defined exercise I. Am. Heart J. 164: 117-124.

16. Kelley GA, Kelley KS. 2009. Impact of progressive resistance training on lipids and lipoproteins in adults: another look at a meta-analysis using prediction intervals. Prev. Med. 49: 473-475.

17. Shaw K, Gennat H, O'Rourke P, Del Mar C. 2006. Exercise for overweight or obesity. Cochrane Database Syst. Rev. 4: CD003817.

18. Brown L, Rosner B, Willett WW, Sacks FM. 1999. Cholesterol-lowering effects of dietary fiber: a meta-analysis. Am. J. Clin. Nutr. 69: 30-42.

19. Gylling H, Plat J, Turley S, Ginsberg HN, Ellegard L, Jessup W, et al. 2014. Plant sterols and plant stanols in the management of dyslipidaemia and prevention of cardiovascular disease. Atherosclerosis 232: 346-360.

20. Hollaender PL, Ross AB, Kristensen M. 2015. Whole-grain and blood lipid changes in apparently healthy adults: a systematic review and meta-analysis of randomized controlled studies. Am. J. Clin. Nutr. 102: 556-572.

21. Musa-Veloso K, Poon TH, Elliot JA, Chung C. 2011. A comparison of the LDL-cholesterol lowering efficacy of plant stanols and plant sterols over a continuous dose range: results of a meta-analysis of randomized, placebocontrolled trials. Prostaglandins Leukot Essent Fatty Acids 85: 9-28.

22. Yu-Poth S, Zhao G, Etherton T, Naglak M, Jonnalagadda S, Kris-Etherton PM. 1999. Effects of the national cholesterol education program's step I and step II dietary intervention programs on cardiovascular disease risk factors: a metaanalysis. Am. J. Clin. Nutr. 69: 632-646.

23. Teh SS, Ahmad R, Wan-Abdullah WN, Liong MT. 2009. Evaluation of agrowastes as immobilizers for probiotics in soy milk. J. Agric. Food Chem. 57: 10187-10198.

24. Shimizu M, Hashiguchi M, Shiga T, Tamura HO, Mochizuki M. 2015. Meta-analysis: effects of probiotic supplementation on lipid profiles in normal to mildly hypercholesterolemic individuals. PLoS One 10: e0139795.

25. Huang Y, Zheng Y. 2010. The probiotic Lactobacillus 
acidophilus reduces cholesterol absorption through the down-regulation of Niemann-Pick C1-like 1 in Caco-2 cells. Br. J. Nutr. 103: 473-478.

26. Huang Y, Wang J, Quan G, Wang X, Yang L, Zhong L. 2014. Lactobacillus acidophilus ATCC 4356 prevents atherosclerosis via inhibition of intestinal cholesterol absorption in apolipoprotein E-knockout mice. Appl. Environ. Microbiol. 80: 7496-7504

27. Huang Y, Wang J, Cheng Y, Zheng Y. 2010. The hypocholesterolaemic effects of Lactobacillus acidophilus American type culture collection 4356 in rats are mediated by the down-regulation of Niemann-Pick C1-like 1. Br. J. Nutr. 104: 807-812.

28. Consonni A, Cordiglieri C, Rinaldi E, Marolda R, Ravanelli I, Guidesi E, et al. 2018. Administration of bifidobacterium and lactobacillus strains modulates experimental myasthenia gravis and experimental encephalomyelitis in Lewis rats. Oncotarget 9: 22269-22287.

29. Gerhard D, Sousa F, Andraus RAC, Pardo PE, Nai GA, Neto HB, et al. 2017. Probiotic therapy reduces inflammation and improves intestinal morphology in rats with induced oral mucositis. Braz. Oral Res. 31: e71.

30. Zheng Y, Lu Y, Wang J, Yang L, Pan C, Huang Y. 2013. Probiotic properties of Lactobacillus strains isolated from Tibetan kefir grains. PLoS One 8: e69868.

31. Biagi E, Nylund L, Candela M, Ostan R, Bucci L, Pini E, et al. 2010. Through ageing, and beyond: gut microbiota and inflammatory status in seniors and centenarians. PLoS One 5: e10667.

32. Kato T, Fukuda S, Fujiwara A, Suda W, Hattori M, Kikuchi J, et al. 2014. Multiple omics uncovers host-gut microbial mutualism during prebiotic fructooligosaccharide supplementation. DNA Res. 21: 469-480.

33. Caporaso JG, Kuczynski J, Stombaugh J, Bittinger K, Bushman FD, Costello EK, et al. 2010. QIIME allows analysis of high-throughput community sequencing data. Nat. Methods 7: 335-336.

34. McDonald D, Price MN, Goodrich J, Nawrocki EP, DeSantis TZ, Probst A, et al. 2012. An improved Greengenes taxonomy with explicit ranks for ecological and evolutionary analyses of bacteria and archaea. ISME J. 6: 610-618.

35. Wang Q, Garrity GM, Tiedje JM, Cole JR. 2007. Naive Bayesian classifier for rapid assignment of rRNA sequences into the new bacterial taxonomy. Appl. Environ. Microbiol. 73: 5261-5267.

36. Keys A. 1984. Serum cholesterol response to dietary cholesterol. Am J Clin Nutr. 40: 351-359.

37. Rudling M. 1992. Hepatic mRNA levels for the LDL receptor and HMG-CoA reductase show coordinate regulation in vivo. J. Lipid. Res. 33: 493-501.

38. Wilson MD, Rudel LL. 1994. Review of cholesterol absorption with emphasis on dietary and biliary cholesterol. J. Lipid. Res. 35: 943-955.
39. Tung YC, Lin $\mathrm{YH}$, Chen HJ, Chou SC, Cheng AC, Kalyanam N, et al. 2016. Piceatannol exerts anti-obesity effects in C57BL/6 mice through modulating adipogenic proteins and gut microbiota. Molecules 21.

40. Russo M, Fabersani E, Abeijon-Mukdsi MC, Ross R, Fontana C, Benitez-Paez A, et al. 2016. Lactobacillus fermentum CRL1446 ameliorates oxidative and metabolic parameters by increasing intestinal feruloyl esterase activity and modulating microbiota in caloric-restricted mice. Nutrients 8.

41. Jasso-Padilla I, Juarez-Flores B, Alvarez-Fuentes G, De la Cruz-Martinez A, Gonzalez-Ramirez J, Moscosa-Santillan M, et al. 2017. Effect of prebiotics of Agave salmiana fed to healthy Wistar rats. J. Sci. Food Agric. 97: 556-563.

42. Xie N, Cui Y, Yin YN, Zhao X, Yang JW, Wang ZG, et al. 2011. Effects of two Lactobacillus strains on lipid metabolism and intestinal microflora in rats fed a high-cholesterol diet. BMC Complement. Altern. Med. 11: 53.

43. Catry E, Pachikian BD, Salazar N, Neyrinck AM, Cani PD, Delzenne NM. 2015. Ezetimibe and simvastatin modulate gut microbiota and expression of genes related to cholesterol metabolism. Life Sci. 132: 77-84.

44. Tomaro-Duchesneau C, Saha S, Malhotra M, Jones ML, Labbe A, Rodes L, et al. 2014. Effect of orally administered L. fermentum NCIMB 5221 on markers of metabolic syndrome: an in vivo analysis using ZDF rats. Appl. Microbiol. Biotechnol. 98: 115-126.

45. Liu Y, Zhao F, Liu J, Wang H, Han X, Zhang Y, et al. 2017. Selection of cholesterol-lowering lactic acid bacteria and its effects on rats fed with high-cholesterol diet. Curr. Microbiol. 74: 623-631.

46. Ejtahed HS, Mohtadi-Nia J, Homayouni-Rad A, Niafar M, Asghari-Jafarabadi M, Mofid V, et al. 2011. Effect of probiotic yogurt containing Lactobacillus acidophilus and Bifidobacterium lactis on lipid profile in individuals with type 2 diabetes mellitus. J. Dairy Sci. 94: 3288-3294.

47. Influence of a probiotic soy product on fecal microbiota and its association with cardiovascular risk factors in an animal model. Lipids Health Dis. 10: 126.

48. Bahekar AA, Singh S, Saha S, Molnar J, Arora R. 2007. The prevalence and incidence of coronary heart disease is significantly increased in periodontitis: a meta-analysis. Am. Heart J. 154: 830-837.

49. Begley M, Hill C, Gahan CG. 2006. Bile salt hydrolase activity in probiotics. Appl. Environ. Microbiol. 72: 1729-1738.

50. Park YH, Kim JG, Shin YW, Kim SH, Whang KY. 2007. Effect of dietary inclusion of Lactobacillus acidophilus ATCC 43121 on cholesterol metabolism in rats. J. Microbiol. Biotechnol. 17: 655-662.

51. Parks DJ, Blanchard SG, Bledsoe RK, Chandra G, Consler TG, Kliewer SA, et al. 1999. Bile acids: natural ligands for an orphan nuclear receptor. Science 284: 1365-1368.

52. Fukushima M, Nakano M, Morii Y, Ohashi T, Fujiwara Y, Sonoyama K. 2000. Hepatic LDL receptor mRNA in rats is 
increased by dietary mushroom (Agaricus bisporus) fiber and sugar beet fiber. J. Nutr. 130: 2151-2156.

53. Gilliland SE, Nelson CR, Maxwell C. 1985. Assimilation of cholesterol by Lactobacillus acidophilus. Appl. Environ. Microbiol. 49: 377-381.

54. Liong MT, Shah NP. 2005. Acid and bile tolerance and cholesterol removal ability of lactobacilli strains. J. Dairy Sci. 88: 55-66.

55. Fei L, Zhang J, Niu H, Yuan C, Ma X. 2016. Effects of rosuvastatin and MiR-126 on myocardial injury induced by acute myocardial infarction in rats: role of vascular endothelial growth factor A (VEGF-A). Med. Sci. Monit. 22: 2324-2334.

56. Kunze A, Huwyler J, Camenisch G, Poller B. 2014. Prediction of organic anion-transporting polypeptide 1B1and 1B3-mediated hepatic uptake of statins based on transporter protein expression and activity data. Drug Metab. Dispos. 42: 1514-1521.

57. Zhao MM, Li D, Li Y. 2015. [Regulation of organic anion transporting polypeptides expression and activity]. Yao Xue Хие Вао 50: 400-405.
58. Le Vee M, Jouan E, Stieger B, Lecureur V, Fardel O. 2011 Regulation of drug transporter expression by oncostatin $\mathrm{M}$ in human hepatocytes. Biochem. Pharmacol. 82: 304-311.

59. Naruszewicz M, Johansson ML, Zapolska-Downar D, Bukowska H. 2002. Effect of Lactobacillus plantarum 299v on cardiovascular disease risk factors in smokers. Am. J. Clin. Nutr. 76: 1249-1255.

60. Wang IK, Wu YY, Yang YF, Ting IW, Lin CC, Yen TH, et al. 2015. The effect of probiotics on serum levels of cytokine and endotoxin in peritoneal dialysis patients: a randomised, double-blind, placebo-controlled trial. Benef. Microbes. 6: 423430 .

61. Karamese M, Aydin H, Sengul E, Gelen V, Sevim C, Ustek D, et al. 2016. The immunostimulatory effect of lactic acid bacteria in a rat model. Iran J. Immunol. 13: 220-228.

62. Martinez I, Wallace G, Zhang C, Legge R, Benson AK, Carr TP, et al. 2009. Diet-induced metabolic improvements in a hamster model of hypercholesterolemia are strongly linked to alterations of the gut microbiota. Appl. Environ. Microbiol. 75: 4175-4184. 